\title{
What Do We Know about the Molecular Mechanisms of Membrane Fusion and Fission?
}

\author{
Yu. A. Chizmadzhev \\ Frumkin Institute of Physical Chemistry and Electrochemistry, Russian Academy of Sciences, Moscow \\ DOI: $10.1134 / \mathrm{S} 1990747809030155$
}

Cell life is accompanied by continuously proceeding events of fusion and fission of various membrane formations. Most of them are very useful for a cell, while others, such as virus entry in the course of endocytosis, may be dangerous. The best example of the important fusion event is exocytosis of synaptic vesicles responsible for neural activity. This issue can justify my fusion/fission talk at this meeting devoted to electrophysiology.

The generic concepts concerning the mechanism of membrane fusion were developed in the course of the experimental and theoretical studies of the remodeling of two planar lipid bilayers. It was shown that fusion consists of the following stages: 1) formation of close intermembrane contact; 2) spontaneous appearance of monolayer bridge known as a stalk, extending in time up to so-called hemifusion diaphragm; 3 ) rupture of the diaphragm leading to a fusion pore opening.
Checking the validity of this mechanism in vivo for cell exocytosis turned out to be very difficult because fusion machine usually includes a lot of different proteins of unknown structure and function. Lucky exclusion is influenza virus-induced fusion governed by a single protein, hemagglutinin, of established structure. As a result, it was proved that fusion in cellular systems is a subject of the same physical principles discovered on lipid models. Studies of SNARE-induced fusion of liposomes have shown that stalk is a most probable intermediate along the pathway of membrane remodeling.

Recently we obtained significant results concerning molecular mechanism of membrane fission. Using new experimental system, a lipid nanotube (NT) made from a planar lipid bilayer by patch pipette in the presence of dynamin and GTP, we observed NT fission and proposed a mechanism based on a quantitative theory. 